\title{
Translation of Human Genome
}

\section{Jun Ling}

Assistant Professor of Biochemistry, Department of Basic Sciences, The Commonwealth Medical College, Scranton, PA 18509, USA

The number of genes ( 23,000 genes) in the human genome is not much larger than those in lower eukaryotes, however the biological functions of human genome are more intricate and diverse due largely to the complex network of regulation of gene expression. The interaction between each step of gene expression, especially at transcriptional and translational levels, contributes to the large human proteome with estimated 1 million proteins for the vast physiological functions of the human body. As important as it is, gene expression profiling has become a routine experimental approach to study various physiological and pathological questions. Yet, most of current techniques rely on the quantitation of mRNA rather than simultaneous measurement of protein production, leading to a gap between mRNA and protein abundance and an inaccurate explanation of biological functions.

The completion of human genome sequencing project in 2003 has shifted the research paradigm from a gene-based approach to a systems biology trend. Consequently, numerous high throughput techniques have been dramatically improved, exemplified by DNA microarray and next-generation sequencing (NGS), which allow us to analyze gene expression at the genome level. Genome-wide gene expression profiling has been carried out for many diseases. The first global map of human gene expression was accomplished [1] with as many as 369 different types of cells, tissues and disease states. Without a doubt, these achievements fundamentally enhanced our understanding of disease mechanisms; however a major piece of information linking a gene to its function is missing- protein expression. Proteins are functional molecules to carry out the biological tasks of genes. Without knowing protein levels, the change in mRNA abundance sometimes cannot explain the phenotype or generates contradictory results. This phenomenon of ignorance of protein synthesis as a part of gene expression is misled by a common sense that gene expression is highly regulated by transcription, whereas mRNA translation is considered as a robust process, which is not true from our current understanding of translational control and regulatory RNAs manifested by the discovery of RNAi and miRNA during the last decade. Technically, a high throughput protein assay is also hindered by the lack of robust technique like RT-PCR, microarray and RNA-seq with NGS. The high dynamics and post-translational modifications of proteins pose additional chemical obstacles for protein analysis. Fortunately, mass spectrometry (MS)-based proteomics has advanced to a level sophisticated enough to identify and quantitate proteins on a genome scale,unveiling proteomes in many diseases and cellular processes [2].

The combination of NGS and MS drives us to revisit the importance of protein synthesis in gene expression. It is noteworthy to mention two major technical advances: one is the "4sU-seq" by NGS that can accurately measure the dynamics of newly synthesized mRNA through pulse labeling with 4-thiouridine at physiological conditions [3]; the other is the "quantitative MS" through stable isotope labeling with amino acids in cell culture (SILAC) or absolute quantification of proteins (AQUA) [4]. The direct measurement of protein in gene expression is also driven by the increasing facts that mRNA levels do not always correlate with protein abundance. Specific studies are now devoted intensively to this topic. In one report, absolute mRNA and protein levels were measured simultaneously in mouse fibroblast using the methods described above, leading to the conclusion that mRNA levels only account for $\sim 40 \%$ of the variation in protein abundance, as it is mostly controlled by mRNA translation [5]. Another study addressed this issue in mouse liver tissue associated with clinical traits. It was discovered that mRNA levels correlated with protein abundance for only about half of the genes tested [6]. More studies have been done in yeast and Arabidopsis [7,8], and all have concluded that the discrepancy between mRNA and protein levels is a common characteristic of gene expression. The concordance varies significantly from species to species and among cellular pathways. Also, it is believed that mRNA-directed mechanisms play a major role in gene expression.

Eukaryotic mRNAs possess diverse sequence signatures that control mRNA processing, localization, stability, and translational . These regulatory cis-elements include, but are not limited to, 5'-Cap, 5'-UTR(untranslated region), IRES(internal ribose entry site), Kozak sequence, 3'-UTR, poly (A) tail and even a regulatory motif within coding region [9]. The last decade has witnessed the fastest growth in our understanding of mRNA translation. It is now recognized that mRNA translation is a critical regulatory step in gene expression [10] and a goal keeper of gene expression fidelity [11]. mRNA translation is more sensitive than gene transcription in response to cell signaling [12], thus provides a more flexible mechanism to regulate gene expression. Some mRNAs from critical genes (e.g., receptors, oncogenes and tumor suppressors, etc.) generally contain IRES and are translated through cap-independent mechanism, showing inconsistence between their mRNA and protein levels. miRNA has emerged as an important regulator of gene expression that functions by controlling mRNA translation and /or degradation. Thus, it is imperative to integrate translational control into the study of gene expression. This trend will also promote the development of bioinformatics tools to better annotate mRNA functional elements.

With the exception of the genome research described above, current studies are still being conducted at gene- or pathway-based levels in most research laboratories. When gene expression profiling is performed, one should be mindful to have their target genes verified not only by qRT-PCR but also by quantitative Western Blotting. If discrepancy arises, the full-length mRNA sequence,including the 5 ' and 3'-UTR should be examined to see if some regulatory cis-elements are involved. The translational activity of the mRNA should be directly measured in translation systems in vivo and in vitro. Polysome profiling

*Corresponding author: Jun Ling, Assistant Professor of Biochemistry, Department of Basic Sciences, The Commonwealth Medical College, Scranton PA 18509, USA, E-mail: jling@tcmedc.org

Received August 08, 2011; Accepted August 10, 2011; Published August 12 2011

Citation: Ling J (2011) Translation of Human Genome. Biochem \& Anal Biochem 1:101e. doi:10.4172/2161-1009.1000101e

Copyright: @ 2011 Ling J. This is an open-access article distributed under the terms of the Creative Commons Attribution License, which permits unrestricted use, distribution, and reproduction in any medium, provided the original author and source are credited. 
is another efficient technique to confirm the translational activity of specific mRNA. Collectively, these strategies will foster a better understanding of the mechanisms that regulate gene expression and the consequence on biological function.

\section{References}

1. Lukk M, Kapushesky M, Nikkilä J, Parkinson H, Goncalves A, et al. (2010) A global map of human gene expression. Nat Biotechnol 28: 322-324.

2. Pontén F, Gry M, Fagerberg L, Lundberg E, Asplund A, et al. (2009) A global view of protein expression in human cells, tissues, and organs. Mol Syst Biol 5: 337.

3. Rabani M, Levin JZ, Fan L, Adiconis X, Raychowdhury R, et al. (2011) Metabolic labeling of RNA uncovers principles of RNA production and degradation dynamics in mammalian cells. Nat Biotechnol 29: 436-442.

4. Gerber SA, Rush J, Stemman O, Kirschner MW, Gygi SP (2003) Absolute quantification of proteins and phosphoproteins from cell lysates by tandem MS. Proc Natl Acad Sci U S A 100: 6940-9645.

5. Schwanhäusser B, Busse D, Li N, Dittmar G, Schuchhardt J, et al. (2011) Global quantification of mammalian gene expression control. Nature 473: 337-342.

6. Ghazalpour A, Bennett B, Petyuk VA, Orozco L, Hagopian R, et al. (2011) Comparative analysis of proteome and transcriptome variation in mouse. PLoS Genet 7: e1001393.

7. Foss EJ, Radulovic D, Shaffer SA, Ruderfer DM, Bedalov A, et al. (2007) Genetic basis of proteome variation in yeast. Nat Genet 39: 1369-1375.

8. Baerenfaller K, Grossmann J, Grobei MA, Hull R, Hirsch-Hoffmann M, et al. (2008) Genome-scale proteomics reveals Arabidopsis thaliana gene models and proteome dynamics. Science 320: 938-941.

9. Ling J, Wells DR, Tanguay RL, Dickey LF, Thompson WF, et al. (2000) Heat shock protein HSP101 binds to the Fed-1 internal light regulatory element and mediates its high translational activity. The Plant Cell 12: 1213-1228.

10. Sonenberg N, Hinnebusch AG (2009) Regulation of translation initiation in eukaryotes: mechanisms and biological targets. Cell 136: 731-745.

11. Zaher HS, Green R (2009) Fidelity at the molecular level: lessons from protein synthesis. Cell 136: 746-762.

12. Ling J, Morley SJ, Traugh JA (2005) Inhibition of cap-dependent translation via phosphorylation of elF4G by protein kinase Pak2. EMBO J 24: 4094-4105. 